\title{
Optical Sky Brightness at Cerro Tololo Inter-American Observatory from 1992 to $2006^{1}$
}

\author{
Kevin Krisciunas, ${ }^{2}$ Dylan R. Semler, ${ }^{3}$ Joseph Richards, ${ }^{4}$ Hugo E. Schwarz, ${ }^{5}$ Nicholas B. \\ Suntzeff, ${ }^{2}$ Sergio Vera,${ }^{6}$ and Pedro Sanhueza ${ }^{7}$
}

\begin{abstract}
We present optical (UBVRI) sky brightness measures from 1992 through 2006. The data are based on CCD imagery obtained with the CTIO 0.9-m, 1.3-m, and 1.5 -m telescopes. The $B$ - and $V$-band data are in reasonable agreement with measurements previously made at Mauna Kea, though on the basis of a small number of images per year there are discrepancies for the years 1992 through 1994. Our CCD-based data are not significantly different than values obtained at Cerro Paranal. We find that the yearly averages of $V$-band sky brightness are best correlated with the $10.7-\mathrm{cm}$ solar flux taken 5 days prior to the sky brightness measures. This implies an average speed of $350 \mathrm{~km} \mathrm{~s}^{-1}$ for the solar wind. While we can measure an enhancement of the night sky levels over La Serena 10 degrees above the horizon, at elevation angles above 45 degrees we find no evidence that the night sky brightness at Cerro Tololo is affected by artificial light of nearby towns and cities.
\end{abstract}

Subject headings: Astronomical Phenomena and Seeing

\footnotetext{
${ }^{1}$ Based in part on observations taken at the Cerro Tololo Inter-American Observatory, National Optical Astronomy Observatory, which is operated by the Association of Universities for Research in Astronomy, Inc. (AURA) under cooperative agreement with the National Science Foundation.

${ }^{2}$ Texas A. \& M. University, Department of Physics, 4242 TAMU, College Station, TX 77843; krisciunas@physics.tamu.edu, suntzeff@physics.tamu.edu

${ }^{3}$ Columbia University, 1027 Pupin Hall MC 5247, 550 W. 120th Street, New York, NY 10027; dsemler@astro.columbia.edu

${ }^{4}$ Department of Statistics, Baker Hall, Carnegie Mellon University, Pittsburgh, PA 15213; joeyrichar@gmail.com

${ }^{5}$ Deceased, 20 October 2006.

${ }^{6}$ Cerro Tololo Inter-American Observatory, Casilla 603, La Serena, Chile

${ }^{7}$ Oficina de Protección de la Calidad del Cielo del Norte de Chile (OPCC), 1606 Cisternas, La Serena, Chile; psanhueza@opcc.cl
} 


\section{Introduction}

A knowledge of the sky background is fundamental to optical and infrared observational astronomy. The accuracy of photometric measurements hinges on the signal-to-noise ratio, so we would like the noise to be as small as possible. The noise has a number of components, among them the dark counts (or dark current), the readout noise, the sky background, and the cosmic ray flux. Furthermore, an instrument must be matched to the typical seeing at a given site, and that stipulates an optimum pixel size for a digital detector. For existing sites and all planned facilities we want to know: 1) the site quality; 2) what kind of natural atmospheric variations there are on short time scales and long time scales; and 3) whether population growth in the area is affecting the astronomical site quality. Another issue we will emphasize in this paper is that of telescope baffling. If a telescope is poorly baffled, then skylight is scattered around the inside of the telescope, raising the background against which we are trying to measure faint astronomical targets. Not much can be done for old telescopes, but this is a critical issue for the design and commissioning of new telescopes.

The literature on the subject of sky brightness is quite large and continues to grow. In particular, the reader is directed to Roach \& Gordon (1973), Leinert et al. (1998), and references therein. Data obtained at specific sites are described by Walker (1988), Pilachowski et al. (1989), Krisciunas (1997, hereafter K97), Benn \& Ellison (1998), Patat (2003), and Patat (2007).

Rayleigh (1928) and Rayleigh \& Jones (1935) were the first to note a possible correlation between the sky brightness and the solar cycle. See Walker (1988) for a more detailed discussion. There are, of course, different measures of solar activity. Walker (1988, Fig. 4) shows a reasonably strong correlation between the $V$-and $B$-band sky brightness as a function of the 10.7-cm solar flux 8 K97, Fig. 3, shows a good correlation of the zenith $V$-band sky brightness vs. the 10.7-cm solar flux if we eliminate data from the years 1985 and 1993 from the analysis. It is not too surprising that there is a correlation of the $V$ band sky brightness and some measure of the solar activity. The solar wind energizes the Earth's upper atmosphere, causing occasional auroral displays. A much smaller effect is the nightly airglow, which has the same origin. The strong atmospheric emission line at 557.7 $\mathrm{nm}$, attributed to $[\mathrm{O} \mathrm{I}]$, falls in the $V$-band 9 While this line contributes directly to $V$-band

\footnotetext{
${ }^{8}$ The units of the solar flux are $10^{-22} / \mathrm{m}^{2} / \mathrm{Hz}$. For this paper we obtained the 10.7 -cm solar flux values from ftp://ftp.ngdc.noaa.gov/STP/SOLAR_DATA/SOLAR_RADIO/FLUX/. These are the "Observed, Series C" data from Penticton, British Columbia.

${ }^{9}$ We note that the Sloan Digital Sky Survey's $g^{\prime}$ and $r^{\prime}$ bands are strategically chosen so that the 557.7 $\mathrm{nm}$ line falls in between their response curves (Fukugita et al. 1996).
} 
sky brightness, the solar flux must also contribute to $B$-band sky brightness variations. K97 found that the color of the sky was quite constant over the course of the solar cycle, with $\langle B-V\rangle=0.930 \pm 0.018$.

In this paper we discuss 15 years of sky brightness measures obtained at Cerro Tololo Inter-American Observatory. The data were obtained with CCD detectors on the CTIO $0.9-\mathrm{m}, 1.3-\mathrm{m}$, and $1.5-\mathrm{m}$ telescopes. The $0.9-\mathrm{m}$ and $1.5-\mathrm{m}$ telescopes were built in the late 1960 's, so are no longer modern telescopes. The 1.3-m telescope was originally used for the Two Micron All Sky Survey (2MASS). Following the conclusion of that survey the 1.3-m was transferred to CTIO, and, along with the 0.9-m, 1.0-m, and 1.5-m telescopes, became part of the Small and Moderate Aperture Research Telescope System (SMARTS) in 2003. Preliminary analysis of the CTIO data was discussed by Vera et al. (2002).

\section{Deriving the Sky Brightness}

Our CCD-based data were reduced within the IRAF 10 environment. First the images are bias-corrected, trimmed, and flattened. The 0.9-m images are typically read out with four amplifiers, which have different effective gains, but the resulting flattened frames show no significant background differences in the four quadrants. To calibrate the sky brightness data on any given night we used 3 to 10 standards of Landolt (1992).

Using DOPнот or DAOPHOT it is possible to determine the point spread function (PSF) of the telescope and CCD camera for every frame, then, using this information, subtract the stars, galaxies, and cosmic rays from the frames. We did not do this. Instead, using some IRAF scripts written by one of us (NBS), we simply made use of the IMHIST program. Since a majority of the pixels are looking at sky, the mode of the pixel counts will correspond to the sky level 11 After iteratively clipping low and high pixels, we fit a Gaussian function to the remainder of the data in the histogram. The peak of this Gaussian fit gives us the most robust value of the number of counts in the sky. Of course, one assumes that the master bias frame and overscan regions used for bias correction remove the bias without the addition of any significant systematic effect. Any problems with bias subtraction can be essentially

\footnotetext{
${ }^{10} \mathrm{IRAF}$ is distributed by the National Optical Astronomy Observatory, which is operated by AURA, Inc. under cooperative agreement with the National Science Foundation.

${ }^{11}$ We carried out tests with imagery of two globular clusters and found that our IRAF scripts gave nearly identical sky brightness values compared to those obtained using IMSTAT on small sub-areas or deriving the median sky counts in a sky annulus while doing aperture photometry on more isolated stars at the edges of the fields.
} 
eliminated by deriving the sky brightness from frames having long exposures (e.g. $300 \mathrm{sec}$ or longer).

Say we perform large aperture photometry on a standard star using APPHOT within IRAF, and this gives us a total of $\mathrm{C}_{\star}$ counts above sky with an exposure time of $\mathrm{E}_{\star}$. The standard star is observed at airmass $\mathrm{X}_{\star}$. The atmospheric extinction in that band (either assumed or derived) is $k_{\lambda}$. Let the standard magnitude of the star from Landolt (1992) be $\mathrm{M}_{\star}$. Let $\mathrm{C}_{s k y}$ be equal to the mean sky counts times the area of the software aperture in a different image with exposure time $\mathrm{E}_{\text {sky }}$. Following Eq. 1 of K97, the magnitude of the sky signal is then

$$
S=-2.5 \log \left(\mathrm{C}_{\mathrm{sky}} / \mathrm{C}_{\star}\right)+2.5 \log \left(\mathrm{E}_{\mathrm{sky}} / \mathrm{E}_{\star}\right)+\mathrm{k}_{\lambda} \mathrm{X}_{\star}+\mathrm{M}_{\star}
$$

One assumes that there are no systematic errors in the exposure times as given by the data acquisition system. Obviously, tests can and should be done to investigate this question. The basic rule is: longer exposures are better.

Since the catalogue value of the standard star magnitude corresponds to its out-ofatmosphere value, one corrects the standard star signal for the extinction in the Earth's atmosphere by adding the term $k_{\lambda} X_{\star}$. The sky brightness along some line of sight in the sky is not corrected to an out-of-atmosphere value. Given the plate scale of the CCD image (i.e., the number of arc seconds per pixel), we can calculate the area of the software aperture A, measured in square arc seconds. The sky brightness $\mathrm{I}(\mu)$ in magnitudes per square arc second is then

$$
I(\mu)=S+2.5 \log \mathrm{A} .
$$

Of course, one can also fit a PSF to the standard stars to obtain the number of counts above sky. The corresponding apparent magnitude of a sky patch can directly be transformed into the sky brightness in magnitudes per square arc second by knowing the plate scale and calculating the area of the sky patch. Finally, one can use measurements of multiple standard stars to give a more robust calibration of the sky flux. Since the sky has the color of a K0type star, one should avoid blue standard stars in order to eliminate as much as possible any filter effects.

Because magnitudes are a logarithmic system, for statistical purposes it is not correct to average sky brightness values in $\mathrm{mag} / \mathrm{sec}^{2}$. One should convert the data to some kind of flux units, average them, then convert the numbers back to mag $/ \mathrm{sec}^{2}$. Following Garstang (1989), Schaefer (1990), and K97, for the $V$ - and B-bands one can use nanoLamberts for the 
flux:

$$
B_{o b s}(\mathrm{~nL})=0.263 \mathrm{a}^{[\mathrm{Q}-\mathrm{I}(\mu)]}
$$

where $a=(100)^{0.2} \approx 2.51189, \mathrm{Q}=10.0+2.5 \log \left(3600^{2}\right) \approx 27.78151, \mathrm{I}(\mu)$ is the sky brightness in $\mathrm{mag} / \mathrm{sec}^{2}$, and the factor 0.263 is the surface brightness (in $\mathrm{nL}$ ) of a star with $V=10$ spread out over one square degree.

For airmass less than 1.6 (and possibly larger) it is appropriate to correct the observed sky brightness to the zenith value using Eq. 1 of Schaefer (1990):

$$
B_{z e n}=B_{o b s} /\left(1+Z_{\text {rad }}^{2} / 2\right)
$$

where $\mathrm{Z}_{\text {rad }}$ is the zenith angle in radians.

As noted above, the CTIO $0.9-\mathrm{m}$ and $1.5-\mathrm{m}$ telescopes are 40 years old. Our analysis shows that the CTIO 1.3-m telescope gives, on average, demonstrably fainter sky brightness values compared to data from the two much older telescopes. After some simple experiments in the dome, we attribute this to bad baffling in the older telescopes. The bottom line is that the camera window facing the Cassegrain secondary mirror should only receive light from that secondary. A poorly baffled telescope will allow light scattering off the inside of a solid telescope tube to hit the CCD camera window. This will brighten the sky background. A poorly baffled telescope with an open tube will allow light from the sky and light from the inside of the dome to degrade the measured sky brightness.

Using imagery obtained with the three telescopes during 2003, 2004, and 2005, we have derived baffle corrections for the data obtained with the $0.9-\mathrm{m}$ and $1.5-\mathrm{m}$ telescopes (Table 11) 12 We assume explicitly that the more modern CTIO 1.3-m telescope is well baffled and the sky brightness values from images obtained with it are correct.

Note that the baffle corrections increase monotonically with wavelength, reaching half a magnitude in the $I$-band. If there were other factors contributing to systematic errors in our CTIO data from 1992 through 2002, it would be difficult to determine at this stage.

Finally, we note that the $U$-band baffling corrections for the older telescopes are inconsistent with the $B V R I$ corrections, in the sense that they equal $-0.27 \mathrm{mag} / \mathrm{sec}^{2}$ for the

\footnotetext{
${ }^{12}$ This is to say that the baffle corrections are adjustments for systematic errors in the $0.9-\mathrm{m}$ and $1.5-\mathrm{m}$ data. These adjustments could have systematic errors of their own, which we estimate to be of order \pm 0.05 $\mathrm{mag} / \mathrm{sec}^{2}$.
} 
0.9-m and $0.00 \mathrm{mag} / \mathrm{sec}^{2}$ for the $1.5-\mathrm{m}$. If bad baffling is the cause of the arithmetically positive corrections for the other filters, then it does not make sense that the CCD camera on the $1.3-\mathrm{m}$ would suffer local light pollution only in the $U$ filter.

\section{A Sanity Check on Systematic Errors}

As a sanity check, we shipped to Chile the photometer and telescope used by K97 for his sky brightness measures obtained at Mauna Kea from 1985 through 1996 (Krisciunas 1996). That system gives an elliptical footprint on the sky of $6.522 \pm 0.184$ square arc minutes and uses an RCA 931A photomultiplier tube. Given the nature of this instrument, it was difficult to avoid stars fainter than $\mathrm{V}=13$ in the beam. Ironically, poor tracking allowed us to sample a small swath of sky and pick off the minimum sky signal. We would expect that CCD-based sky brightness values would be somewhat fainter than data obtained with the Krisciunas system, since faint stars and galaxies can be eliminated from CCD analysis.

In Tables 2 and 3 we give some sky brightness values obtained on two photometric nights at CTIO in December of 2006 13 Table 2 gives data obtained with the CTIO 0.9-m telescope. Some of the $V$-band sky brightness values were obtained within two hours of the end of astronomical twilight (which occurred at roughly 01:16 UT on those nights). The other CCD data were obtained at a fixed location on the sky, RA $=5$ hours, DEC $=-30$ degrees. Table 3 gives data obtained with the Krisciunas system at a number of positions west of the celestial meridian on the very same nights.

Fig. 1 shows the sky brightness measures obtained at CTIO with the two different systems on 2006 December 23 and 24 UT. Clearly, there is evidence that the sky continued to get darker long after the nominal end of astronomical twilight. We shall consider only the data obtained more than two hours after the end of astronomical twilight. In the $V$ - and $B$-bands, respectively, the data from the Krisciunas system are, on average, 0.13 and 0.17 $\mathrm{mag} / \mathrm{sec}^{2}$ brighter than the baffle-corrected 0.9 -m data. These differences can be attributed to a combination of factors: 1) uncertainty in the beam size of the Krisciunas system; 2) the unknown contribution of faint stars in the Krisciunas system beam; and 3) systematic

\footnotetext{
${ }^{13}$ Landolt (1992) fields were observed in BVRI on 7 occasions over the course of 2006 December 23 and 24. Using EVALFIT within the PHOTCAL package, we found that the RMS uncertainties of the BVRI magnitudes of the standards were between \pm 0.01 and $\pm 0.02 \mathrm{mag}$ on these nights. Extinction values were measured to $\pm 0.01 \mathrm{mag} /$ airmass. Thus, we judge these two nights to be of excellent photometric quality. For the calibration of the single channel photometer data our principal standard stars were BS 1179 and $\zeta$ Cae. Our check star was $\rho$ For. Their $B$ and $V$ magnitudes were obtained from Hoffleit \& Jaschek (1982).
} 
errors in the baffling corrections for the $0.9-\mathrm{m}$. On the whole, however, the data obtained with the Krisciunas system and the $0.9-\mathrm{m}$ are in reasonable agreement because one would expect the single channel photomultipler tube data to give brighter values than CCD data based on pixels that were free of the light of stars and galaxies.

We note that the recent data obtained with the Krisciunas system (corrected to the zenith) give $\langle B-V\rangle=0.906 \pm 0.034$, while the data from the 0.9 -m obtained on the same two nights (and more than two hours after the end of astronomical twilight) give $\langle B-V\rangle=0.951 \pm 0.013$. These values are in good agreement with the average from K97 of $\langle B-V\rangle=0.930 \pm 0.018$.

On 2006 December 23 and 24 we also measured the sky brightness at 10 to 11 degrees above the left flank of La Serena 14 In Figs. 4 and 5 of Garstang (1989) we find the results of his modelling the atmosphere at Boulder, Colorado (elevation $1655 \mathrm{~m}$ ), and Mt. Graham, Arizona (elevation $3267 \mathrm{~m}$ ) 15 Since CTIO is $2215 \mathrm{~m}$ above sea level, it makes sense to average the two models for our purposes here. We note, however, that the continental air of the United States is not as aerosol-free as the maritime air of CTIO. We assume that the total contribution to the $V$-band sky brightness from directly transmitted light, Rayleigh scattering, and aerosol scattering is 1.94 times brighter at a zenith angle of 79 or 80 degrees compared to the contribution at the zenith. For Garstang's Boulder model the value is 1.84 and for Mt. Graham the value is 2.03. In Table 4 we convert some of our data from Table 3 to fluxes in $\mathrm{nL}$ and compare the observed fluxes at high airmass with what we would predict on the basis of the mean zenith sky brightness scaled by the factor from Garstang's model.

Luginbuhl (2007, private communication) indicates that on one recent occasion he and his colleagues measured the sky brightness near Flagstaff, Arizona, to be $V=21.85 \mathrm{mag} / \mathrm{sec}^{2}$ at the zenith and $21.21 \mathrm{mag} / \mathrm{sec}^{2}$ at elevation angle 10 degrees. Those numbers translate into a flux ratio of 1.80 . Whether for CTIO the most robust value of this parameter is 1.8 or 2.0 , we observed $\approx 3$ times as much flux at high zenith angle compared to the zenith.

From the summit of Cerro Tololo one can look down at La Serena, Vicuña, and Andacollo and see artificial light with the naked eye if those locations are not covered by cloud. Statistically speaking, we obtained the same values of the sky brightness at very high airmass on December 23 and 24. At 10 to 11 degrees above the left flank of La Serena we measured enhancements of 72 and 44 percent in the $V$-band on the two nights in question. These are

\footnotetext{
${ }^{14}$ We could not aim directly over the center of the city because the dome of the $0.9-\mathrm{m}$ telescope was in the way.

${ }^{15} \mathrm{~A}$ careful reading of the text of Garstang's paper reveals that the captions to his Figs. 4 and 5 should be swapped.
} 
almost certainly measurements of light pollution attributable to La Serena. At elevation angles of 45 degrees or higher there is no measurable effect on the night sky brightness at Tololo at this time.

\section{A Database of Useful CTIO Sky Brightness Measures}

Over the course of years of observing galaxies that have hosted supernovae, we have accumulated many images. These images can be used for the measurement of the sky brightness at Cerro Tololo. Of course, these images were taken under a variety of sky conditions: photometric, non-photometric, with and without moonlight. Some are short exposures. Some are long exposures. Some were taken during twilight or when the zodiacal light was still strong. Some were taken in the middle of the night.

Our database of images usable for measurement of the sky brightness involved an extensive selection process to reduce the effects of artifical brighteners of the sky. This includes:

1. Removal of images with exposure times shorter than 10 seconds. Given the huge number of pixels in a CCD chip, we find that it is possible to get reliable sky brightness readings with exposures as short as 10 seconds.

2. Removal of images with airmass greater than 1.6. The effect of dust and particles in the Earth's atmosphere begins to dominate the sky brightness levels closer to the horizon. See Garstang (1989, Figs. 4, 5). Limiting the study to low airmasses reduces the effect of these particles on the sky brightness values.

3. Removal of images taken within 30 degrees of the Galactic plane. Any image of the night sky will contain countless unresolved sources which brighten the level of the sky. By excluding images taken in the Galactic plane we significantly decrease the number of unresolved stars that could contribute to this brightening.

4. We include only images taken more than two hours after the end of evening astronomical twilight (i.e. Sun 18 degrees below the horizon) until two hours before the start of morning astronomical twilight. During astronomical twilight the sky is being brightened by the Sun. Up to two hours after the end of evening astronomical twilight and starting two hours before the start of morning astronomical twilight the sky is partially illuminated by the zodiacal light, which is sunlight scattering off interplanetary dust.

5. Removal of images taken when the Moon was above the horizon or if the Moon was within 30 minutes of the horizon. 
6. Removal of images taken on non-photometric nights. Any clouds would have a significant impact on the observed brightness levels. We consulted the historic sky conditions from the CTIO website and excluded nights known to be non-photometric.

7. Removal of images more than three standard deviations from the mean on those nights when multiple images were obtained.

8. For reasons outlined above, we choose to consider only $U$-band values obtained with the CTIO 1.3-m telescope.

In Table 5 we give the yearly averages of the BVRI sky brightness at CTIO. Many of these yearly averages, especially during the 1990's, are based on a small number of images per year. Of course, many other observers were using the CTIO 0.9-m and 1.5-m telescopes. We should have organized a system whereby observers could copy to disk deep images obtained in the middle of the night, along with images of standard stars. The Paranal database described by Patat (2007) is understandably more extensive than ours described here.

As mentioned above, it is not correct to average data in magnitudes or mag $/ \mathrm{sec}^{2}$ because those are logarithmic units. One should convert to fluxes, average the fluxes, and then convert the average back to magnitude units if one so chooses. This is what we have done in our analysis.

Fig. 2 shows the individual zenith $V$-band sky brightness values derived from CCD imagery obtained at CTIO. While a solar cycle effect is apparent, we feel that yearly averages show the effect more clearly.

Fig. 3 shows the yearly averages from K97 along with the CTIO yearly averages. There is an overlap of four years. As first reported by Vera et al. (2002), the CTIO data of 1992 to 1994 are noticeably fainter than the data obtained at the 2800-m level of Mauna Kea and reported by K97. Even if we correct the Mauna Kea data of 1992 for the difference of solar flux levels of the nights in question, we cannot reconcile the numbers. The CTIO $V$-band data of 1992 are based on 3 nights, so we could just be dealing with small number statistics. Perhaps the baffle corrections obtained from imagery of 2003 to 2005 are not the correct values to apply to the data of 1992 through 1994. The small amount of data obtained in 1996 at the two locations matches within the errors, and the sanity check described in $\S 3$ of this paper is reasonable assurance that under careful conditions we get comparable values with the single channel system and the CCD camera on the 0.9-m at CTIO.

In Fig. 4 we show the yearly averages of the BRI sky brightness at Mauna Kea and at CTIO. The Mauna Kea $B$-band data alone show a solar cycle effect, as does the CTIO $B$-band dataset taken on its own. However, as in the $V$-band, there is a serious discrepancy 
as to zeropoint in the years 1992 to 1994 . We see no evidence for a solar cycle effect in the $R$ - and $I$-band data from CTIO.

Grand averages of CTIO and Paranal data are given in Table 6. The Paranal data are based on images taken from April 2001 through April 2006 (Patat 2007). Thus, both datasets cover years of solar maximum and solar minimum. However, the years 2001 through 2006 are not equally represented in the Paranal data. There are more observations from 2001 to 2003 when the Sun was more active. Patat (2007, private communication) indicates that the long term $B$ - and $V$-band sky brightness at Paranal is roughly $0.1 \mathrm{mag} / \mathrm{sec}^{2}$ fainter than the values in Table 6 .

In Table 6 the uncertainties given are the standard deviations of the distributions, not the standard deviations of the means. Statistically speaking, the Paranal data and the CTIO data are in agreement, given the typical standard deviations of $\pm 0.20 \mathrm{mag} / \mathrm{sec}^{2}$. With the $0.1 \mathrm{mag} / \mathrm{sec}^{2}$ adjustment mentioned above, the CTIO data are, on average, $0.06 \mathrm{mag} / \mathrm{sec}^{2}$ fainter than Paranal in $B$ but equal in $V$. This is evidence that our baffling corrections are close to being correct, for these bands at least.

Under the reasonable assumption of a physical cause and effect between activity on the Sun and the chemical reactions occurring in the Earth's atmosphere which result in the airglow, we naturally ask: is this due to the light which shines on the Earth eight minutes after leaving the Sun's photosphere? Or is it due to the solar wind, i.e. to particles coming from the Sun?

In Fig. 5 we plot the yearly averages from Table 5, converted to flux, vs. the mean of the 10.7-m solar flux 4.5 days prior to when the sky brightness was measured. We made various versions of this plot using solar flux values from the day prior to a given night's observations until 8 days prior. Since the solar flux is measured about $0.5 \mathrm{~d}$ prior to a given night's observations, this corresponds to $-8.5 \leq \Delta \mathrm{T} \leq-0.5 \mathrm{~d}$. We find a minimum reduced $\chi^{2}$ value at $\Delta \mathrm{T}=-5.0 \mathrm{~d}$. Given the mean distance of the Earth from the Sun, a time delay of 5.0 days corresponds to a mean speed of the solar wind of $\approx 350 \mathrm{~km} \mathrm{~s}^{-1}$.

This can be compared to the escape speed at the surface of the Sun, $618 \mathrm{~km} \mathrm{~s}^{-1}$, and to the speeds of the leading edges of coronal mass ejections, namely $450 \mathrm{~km} \mathrm{~s}^{-1}$ at solar maximum, and $160 \mathrm{~km} \mathrm{~s}^{-1}$ at solar minimum (Kahler 2000). More extensive photometry and sky spectra obtained at Paranal may shed light on this time delay effect 16

As shown by Walker (1988), Pilachowski et al. (1989), and K97, on any given night the

\footnotetext{
${ }^{16}$ From http://solarscience.msfc.nasa.gov/SolarWind.shtml we can see a graph of the solar wind velocity over the previous seven days. A mean speed of $400 \mathrm{~km} \mathrm{~s}^{-1}$ is quoted, with a range of 300 to $800 \mathrm{~km} \mathrm{~s}^{-1}$.
} 
sky brightness can vary 10 to 50 percent. There is not one single value for any given night. Whole-night wide-angle digital movies of the sky at CTIO obtained by Roger Smith show bands of $\mathrm{OH}$ emission passing over the summit on time scales of tens of minutes. It is not surprising to measure variations of the airglow component of the sky brightness.

\section{Discussion}

Photometry of astronomical point sources in sparse fields is easy. Photometry of stars in crowded fields is more difficult. Photometry of extended sources is much more difficult because one must worry about seeing, contrast against the sky, and plate scale. Photometry of the night sky is of intermediate difficulty. The biggest systematic uncertainties arise from certain aspects of CCD observing that we normally do not worry about: accuracy of exposure times, imperfect bias subtraction, light leaks, and bad baffling in the telescopes.

Ideally, one would like to be able to measure large solid angles of the sky and to calibrate the observed sky brightness by means of many identifiable standard stars. Such a system

has been implemented, and is described by Duriscoe, Luginbuhl, \& Moore (2007). These authors are able to image the entire sky over a span of half an hour and can obtain robust photometric zeropoints and extinctions from the identification and detection of over 100 bright standard stars in each dataset.

A comparison of sky brightness obtained with different equipment is largely a search for systematic errors. Because of the importance of northern Chile to ground based observational astronomy, we felt it was important to calibrate the night sky at Cerro Tololo using images easily available to us. This also involved taking data with the very same telescope and photometer used by Krisciunas (1997) for an 11 year study at Mauna Kea. We find that observations obtained at CTIO with the Krisciunas system are consistent with observations obtained with the CTIO 0.9-m telescope if we adopt corrections for bad baffling in that telescope.

We have used an extensive database of images obtained for supernova research and have whittled down the size of the database by excluding observations on non-photometric nights, observations taken within two hours of the end or beginning of astronomical twilight, observations when the Moon was within 30 minutes of the horizon, images obtained within 30 degrees of the Galactic plane, and images taken at airmass greater than 1.6. The resulting database demonstrates a correlation of the $V$-band sky brightness with the phase of the solar cycle, as has been found by others over the past 80 years. A solar cycle effect can be seen to a lesser extent in the $B$-band data, but there appears to be no significant solar cycle effect 
in the $R$ - and $I$-band data.

We find that the $V$-band sky brightness is most tightly correlated with the solar flux obtained 5 days prior to the night in question. This corresponds to a mean speed of $\approx 350$

$\mathrm{km} \mathrm{s}^{-1}$ for the solar wind, in the mid-range of velocities of coronal mass ejections at solar minimum and solar maximum.

We find no evidence of light pollution at Cerro Tololo within 45 degrees of the zenith at this time. However, 10 degrees over La Serena we measured a $58 \pm 14$ percent enchancement of the $V$-band sky brightness on two nights.

The CTIO 0.9-m, 1.3-m, and 1.5-m telescopes are operated by the Small and Moderate Aperture Research Telescope System (SMARTS) Consortium. DRS is grateful for the opportunity to participate in the Research Experience for Undergraduates (REU) Program of the National Science Foundation. JR thanks the Fulbright U.S. Student Program, IIE (The Institute of International Education). SV is grateful to the Práctica de Investigacíon en Astronomía (PIA) Program. We thank Ferdinando Patat for making data available ahead of publication, and thank Chris Luginbuhl for useful discussions. We particularly thank Malcolm Smith for his encouragement and support of this work. This work was supported by Cerro Tololo Observatory. An obituary of Hugo Schwarz will be published in an upcoming issue of the Bulletin of the American Astronomical Society. Other personal recollections can be found at http://www.subjectivelens.com/Hugo/.

\section{REFERENCES}

Benn, C. R., \& Ellison, S. L. 1998, New Astron. Reviews, 42, 503

Duriscoe, D. M., Luginbuhl, C. B., \& Moore, C. A. 2007, PASP, 119, 192

Fukugita, M., Ichikawa, T., Gunn, J. E., Doi, M., Shimasaku, K., \& Schneider, D. P. 1996, AJ, 111, 1748

Garstang, R. H. 1989, PASP, 101, 306

Hoffleit, D., \& Jaschek, C. 1982, The Bright Star Catalogue, 4th ed. (New Haven, Yale Univ. Obs.)

Kahler, S. 2000, in Allen's Astrophysical Quantities, 4th ed., A. N. Cox, ed. (New York, Springer), 373 
Krisciunas, K. 1996, S\&T, 91, No. 2, 91

Krisciunas, K. 1997, PASP, 109, 1181

Landolt, A. U. 1992, AJ, 104, 340

Leinert, Ch., et al. 1998, A\&AS, 127, 1

Patat, F. 2003, A\&A, 400, 1183

Patat, F. 2007, A\&A, in press

Pilachowski, C. A., Africano, J. L., Goodrich, B. D., \& Binkert, W. S. 1989, PASP, 101, 707

Rayleigh, L. (Strutt, R. J.) 1928, Proc. Roy. Soc. London, Ser. A., 119, 11

Rayleigh, L. (Strutt, R. J.), \& Jones, H. S. 1935, Proc. Roy. Soc. London, Ser. A., 151, 22

Roach, F. E., \& Gordon, J. L. 1973, The Light of the Night Sky (Dordrecht, D. Reidel)

Schaefer, B. E. 1990, PASP, 102, 212

Vera, S., Candia, P., Krisciunas, K., Leiton, R., \& Suntzeff, N. B. 2002, BAAS, 34, 1226

Walker, M. F. 1988, PASP, 100, 496 
Table 1. Baffle Corrections ${ }^{\mathrm{a}}$

\begin{tabular}{ccccc}
\hline \hline Telescope & $B$ & $V$ & $R$ & $I$ \\
\hline $0.9-\mathrm{m}$ & 0.269 & 0.274 & 0.369 & 0.521 \\
$1.5-\mathrm{m}$ & 0.132 & 0.286 & 0.322 & 0.527 \\
\hline
\end{tabular}

${ }^{a}$ The values in the table are the number of magnitudes per square arc second to $a d d$ to the raw sky brightness data to eliminate systematic differences in sky brightness compared to the CTIO 1.3-m telescope. These values are based on data taken during the years 2003 through 2005 . 
Table 2. Sky Brightness Values from CTIO 0.9-m Imagery ${ }^{\mathrm{a}}$

\begin{tabular}{|c|c|c|c|c|c|c|c|c|}
\hline UT Date & $\langle\mathrm{UT}\rangle$ & RA & DEC & Filter & Exptime & Observed & Z & Corrected \\
\hline Dec 23 & 01:07 & $23: 36: 39$ & $-10^{\circ} 15^{\prime}$ & $V$ & 300 & 21.262 & 44.83 & 21.552 \\
\hline Dec 23 & $01: 35$ & 00:14:08 & -1025 & $V$ & 300 & 21.591 & 42.80 & 21.858 \\
\hline Dec 23 & 02:06 & 02:08:18 & -350 & $V$ & 300 & 21.933 & 32.50 & 22.095 \\
\hline Dec 23 & 03:00 & $02: 20: 38$ & -754 & $V$ & 300 & 21.948 & 36.15 & 22.145 \\
\hline Dec 23 & 04:08 & 05:00:00 & -3000 & $V$ & 400 & 22.160 & 6.58 & 22.167 \\
\hline Dec 23 & $04: 42$ & 05:00:00 & -3000 & $V$ & 400 & 22.171 & 15.90 & 22.212 \\
\hline Dec 23 & 05:05 & 05:00:00 & -3000 & $V$ & 400 & 22.124 & 18.83 & 22.181 \\
\hline Dec 23 & 04:00 & 05:00:00 & -3000 & $B$ & 600 & 23.081 & 4.91 & 23.085 \\
\hline Dec 23 & $04: 34$ & 05:00:00 & -3000 & $B$ & 600 & 23.072 & 12.23 & 23.096 \\
\hline Dec 23 & $04: 56$ & 05:00:00 & -3000 & $B$ & 600 & 23.053 & 17.16 & 23.101 \\
\hline Dec 24 & 01:05 & $23: 29: 44$ & -937 & $V$ & 300 & 20.857 & 47.05 & 21.173 \\
\hline Dec 24 & $01: 31$ & $00: 28: 38$ & +021 & $V$ & 300 & 21.241 & 46.75 & 21.553 \\
\hline Dec 24 & $01: 58$ & $02: 20: 37$ & -924 & $V$ & 300 & 21.742 & 25.58 & 21.845 \\
\hline Dec 24 & $02: 54$ & 02:08:18 & -350 & $V$ & 300 & 21.503 & 40.92 & 21.750 \\
\hline Dec 24 & $03: 53$ & 05:00:00 & -3000 & $V$ & 400 & 21.939 & 4.16 & 21.942 \\
\hline Dec 24 & $04: 13$ & 05:00:00 & -3000 & $V$ & 400 & 21.971 & 8.35 & 21.982 \\
\hline Dec 24 & 04:32 & 05:00:00 & -3000 & $V$ & 400 & 22.012 & 12.49 & 22.037 \\
\hline Dec 24 & $04: 53$ & 05:00:00 & -3000 & $V$ & 400 & 22.045 & 17.08 & 22.092 \\
\hline Dec 24 & 05:11 & 05:00:00 & -3000 & $V$ & 400 & 22.011 & 20.99 & 22.082 \\
\hline Dec 24 & $05: 30$ & 05:00:00 & -3000 & $V$ & 400 & 21.977 & 24.94 & 22.075 \\
\hline Dec 24 & $05: 49$ & 05:00:00 & -3000 & $V$ & 400 & 21.960 & 29.12 & 22.092 \\
\hline Dec 24 & 06:07 & 05:00:00 & -3000 & $V$ & 400 & 21.920 & 33.03 & 22.087 \\
\hline Dec 24 & $03: 44$ & 05:00:00 & -3000 & $B$ & 600 & 22.991 & 2.49 & 22.992 \\
\hline Dec 24 & 04:04 & 05:00:00 & -3000 & $B$ & 600 & 22.898 & 6.68 & 22.905 \\
\hline Dec 24 & $04: 23$ & 05:00:00 & -3000 & $B$ & 600 & 22.952 & 10.81 & 22.990 \\
\hline Dec 24 & $04: 44$ & 05:00:00 & -3000 & $B$ & 600 & 22.978 & 15.42 & 23.017 \\
\hline Dec 24 & 05:02 & 05:00:00 & -3000 & $B$ & 600 & 22.991 & 19.32 & 23.051 \\
\hline Dec 24 & $05: 21$ & 05:00:00 & -3000 & $B$ & 600 & 22.968 & 23.28 & 23.054 \\
\hline Dec 24 & $05: 40$ & 05:00:00 & -3000 & $B$ & 600 & 22.937 & 27.46 & 23.055 \\
\hline Dec 24 & $05: 58$ & 05:00:00 & -3000 & $B$ & 600 & 22.916 & 31.38 & 23.068 \\
\hline
\end{tabular}

${ }^{a}$ Year is 2006. UT is in hours and minutes. Right ascension is in hours, minutes, seconds (J2000). Declination is in degrees and arc minutes. Exposure times are in seconds. Column 7 is observed sky brightness in mag $/ \mathrm{sec}^{2}$, using baffling corrections from Table $1 \mathrm{Z}$ is the zenith angle in degrees. Column 9 data in $\mathrm{mag} / \mathrm{sec}^{2}$ are values from column 7 , corrected to the zenith using Eq. 4. 
Table 3. CTIO Sky Brightness Values from Single Channel System ${ }^{a}$

\begin{tabular}{cccccccc}
\hline \hline UT Date & $\langle$ UT $\rangle$ & RA & DEC & Filter & Observed & Z & Corrected \\
\hline Dec 23 & $04: 28$ & $04: 40$ & -30 & $V$ & $22.038(0.06)$ & 15.34 & $22.076(0.06)$ \\
Dec 23 & $04: 47$ & $01: 50$ & +18 & $V$ & $20.761(0.04)$ & 78.54 & $\ldots$ \\
Dec 23 & $05: 15$ & $05: 00$ & -30 & $V$ & $21.992(0.06)$ & 21.18 & $22.064(0.06)$ \\
& & & & & & & \\
Dec 23 & $04: 38$ & $04: 40$ & -30 & $B$ & $22.893(0.10)$ & 17.50 & $22.943(0.10)$ \\
Dec 23 & $04: 45$ & $01: 50$ & +18 & $B$ & $22.266(0.06)$ & 78.92 & $\ldots$ \\
Dec 23 & $05: 18$ & $05: 00$ & -30 & $B$ & $22.884(0.10)$ & 21.83 & $22.960(0.10)$ \\
& & & & & & & \\
Dec 24 & $05: 00$ & $05: 00$ & -30 & $V$ & $21.747(0.06)$ & 18.79 & $21.804(0.06)$ \\
Dec 24 & $05: 10$ & $02: 10$ & +17 & $V$ & $20.782(0.04)$ & 79.67 & $\ldots$ \\
Dec 24 & $05: 24$ & $04: 12$ & -5 & $V$ & $21.657(0.06)$ & 45.11 & $21.950(0.06)$ \\
Dec 24 & $05: 47$ & $05: 00$ & -30 & $V$ & $21.801(0.06)$ & 28.91 & $21.951(0.06)$ \\
& & & & & & & \\
Dec 24 & $05: 00$ & $05: 00$ & -30 & $B$ & $22.750(0.10)$ & 18.79 & $22.807(0.10)$ \\
Dec 24 & $05: 12$ & $02: 10$ & +17 & $B$ & $22.290(0.06)$ & 80.06 & $\ldots$ \\
Dec 24 & $05: 22$ & $04: 12$ & -5 & $B$ & $22.618(0.10)$ & 44.72 & $22.907(0.10)$ \\
Dec 24 & $05: 44$ & $05: 00$ & -30 & $B$ & $22.634(0.10)$ & 28.27 & $22.759(0.10)$ \\
\hline
\end{tabular}

${ }^{a}$ Year is 2006. UT and right ascension are in hours and minutes. Declination is in degrees. Column 6 is observed sky brightness in $\mathrm{mag} / \mathrm{sec}^{2} . \mathrm{Z}$ is the zenith angle in degrees. Column 8 data in $\mathrm{mag} / \mathrm{sec}^{2}$ are values from column 6 , corrected to the zenith using Eq. 4. Values in parentheses are estimated random errors. 
Table 4. Detection of Artificial Light at High Zenith Angle ${ }^{a}$

\begin{tabular}{cccrccc}
\hline \hline UT Date & Filter & $B_{z e n}(\mathrm{~nL})$ & $B_{\text {obs }}(\mathrm{nL})$ & Ratio(obs/zen) & $B_{\text {pred }}(\mathrm{nL})$ & Ratio(obs/pred) \\
\hline Dec 23 & $V$ & 50.7 & 169.1 & 3.34 & 98.4 & 1.72 \\
Dec 23 & $B$ & 22.5 & 42.3 & 1.88 & $\cdots$ & $\cdots$ \\
Dec 24 & $V$ & 59.6 & 165.9 & 2.78 & 115.6 & 1.44 \\
Dec 24 & $B$ & 25.3 & 41.4 & 1.64 & $\cdots$ & $\cdots$ \\
\hline
\end{tabular}

${ }^{\text {a }}$ Year is 2006. The values in column 6 are equal to the values in column 3 times 1.94. This scaling factor is obtained from averaging models of one lower elevation site and one higher elevation site from Figs. 4 and 5 of Garstang (1989) and corresponds to a zenith angle of 79 to 80 degrees.

Table 5. Yearly Averages of Sky Brightness at $\mathrm{CTIO}^{\mathrm{a}}$

\begin{tabular}{ccccccccc}
\hline \hline Year & $\langle B\rangle$ & $\mathrm{N}_{B}$ & $\langle V\rangle$ & $\mathrm{N}_{V}$ & $\langle R\rangle$ & $\mathrm{N}_{R}$ & $\langle I\rangle$ & $\mathrm{N}_{I}$ \\
\hline 1992 & $22.971(0.024)$ & 3 & $21.842(0.056)$ & 5 & $\ldots$ & $\ldots$ & $\ldots$ & $\ldots$ \\
1993 & $23.122(0.085)$ & 2 & 21.897 & 1 & $\ldots$ & $\ldots$ & $\ldots$ & $\ldots$ \\
1994 & $23.259(0.022)$ & 5 & $22.034(0.012)$ & 4 & $\ldots$ & $\ldots$ & $\ldots$ & $\ldots$ \\
1996 & $22.964(0.050)$ & 2 & $21.904(0.005)$ & 2 & $\ldots$ & $\ldots$ & 19.956 & 1 \\
1997 & $22.745(0.057)$ & 11 & $21.803(0.051)$ & 15 & $\ldots$ & $\ldots$ & $\ldots$ & $\ldots$ \\
1998 & $22.982(0.074)$ & 3 & $21.911(0.018)$ & 4 & $\ldots$ & $\ldots$ & $\ldots$ & $\ldots$ \\
1999 & $22.741(0.018)$ & 13 & $21.600(0.039)$ & 11 & $\ldots$ & $\ldots$ & $\ldots$ & $\ldots$ \\
2000 & $22.766(0.048)$ & 8 & $21.564(0.055)$ & 11 & 20.880 & 1 & 19.374 & 1 \\
2001 & $22.870(0.024)$ & 11 & $21.668(0.052)$ & 15 & $21.110(0.190)$ & 3 & $19.828(0.172)$ & 5 \\
2002 & $22.676(0.067)$ & 10 & $21.694(0.045)$ & 13 & $21.162(0.067)$ & 13 & $19.895(0.061)$ & 18 \\
2003 & $22.815(0.028)$ & 42 & $21.817(0.020)$ & 78 & $21.208(0.018)$ & 65 & $19.814(0.032)$ & 71 \\
2004 & $22.772(0.025)$ & 49 & $21.710(0.024)$ & 63 & $21.085(0.019)$ & 60 & $19.848(0.035)$ & 57 \\
2005 & $22.834(0.026)$ & 38 & $21.854(0.024)$ & 95 & $21.278(0.022)$ & 83 & $19.866(0.021)$ & 95 \\
2006 & $22.994(0.032)$ & 13 & $22.061(0.031)$ & 12 & $21.018(0.003)$ & 2 & $19.726(0.032)$ & 2 \\
\hline
\end{tabular}

${ }^{a}$ Values are measured in mag/ $/ \mathrm{sec}^{2}$. The numbers in parentheses are 1- $\sigma$ uncertainties (mean errors of the mean). There are no data from 1995. $\mathrm{N}_{i}$ is the number of images, not the number of nights. 
Table 6. Mean Sky Brightness at CTIO and Paranal ${ }^{\mathrm{a}}$

\begin{tabular}{ccccccccccccc}
\hline \hline Site & $\langle U\rangle$ & $\mathrm{N}_{U}$ & $\langle B\rangle$ & $\mathrm{N}_{B}$ & $\langle V\rangle$ & $\mathrm{N}_{V}$ & $\langle R\rangle$ & $\mathrm{N}_{R}$ & $\langle I\rangle$ & $\mathrm{N}_{I}$ \\
\hline CTIO & $22.12(0.19)$ & 27 & $22.82(0.19)$ & 210 & $21.79(0.22)$ & 329 & $21.19(0.19)$ & 227 & $19.85(0.25)$ & 250 \\
Paranal & $22.35(0.19)$ & 261 & $22.66(0.16)$ & 1332 & $21.69(0.21)$ & 1619 & $20.91(0.23)$ & 3595 & $19.65(0.28)$ & 2882 \\
difference & -0.23 & $\ldots$ & 0.16 & $\ldots$ & 0.10 & $\ldots$ & 0.28 & $\ldots$ & 0.20 & $\ldots$ & $\cdots$ \\
\hline
\end{tabular}

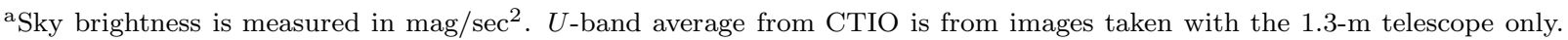
Paranal values from Patat (2007) are based on data from April 2001 through April 2006, but the Paranal averages are weighted more toward 2001 to 2003, when the solar cycle was closer to maximum. The values in parentheses are the standard deviations of the distributions, not the standard deviations of the means. 
Fig. 1.- Values of sky brightness from CTIO. Top panels: $V$-band sky brightness. Bottom panels: $B$-band sky brightness. Left panels: data of 2006 December 23 UT. Right panels: data of 2006 December 24 UT. The blue dots are data from the CTIO 0.9-m reflector. The green squares are data obtained with the single channel system of Krisciunas (1996). All data have been corrected to the zenith.

Fig. 2.- Upper panel: Individual CCD-based values of zenith $V$-band sky brightness from CTIO. Lower panel: 10.7-cm solar flux.

Fig. 3.- Upper panel: Data obtained at the 2800-m level of Mauna Kea using a 15-cm telescope and single channel photometer (Krisciunas 1996), along with the average of the data obtained at CTIO in December of 2006 (green squares). These data were typically taken within 20 degrees of the zenith. The blue dots are yearly averages of data obtained at CTIO using CCD imagery. The CTIO data and the single channel data from December 2006 have all been reduced to the zenith. Lower panel: 10.7-cm solar flux.

Fig. 4.- Yearly averages of BRI sky brightness. In the top panel the squares represent data from K97, along with the data from Table 3 of this paper. Circles represent CCD-based data from CTIO presented in this paper.

Fig. 5.- Yearly averages of zenith $V$-band sky brightness obtained from CCD imagery at Cerro Tololo (converted to flux) vs. the average of the $10.7-\mathrm{cm}$ solar flux 4.5 days prior to when the sky brightness was measured. 

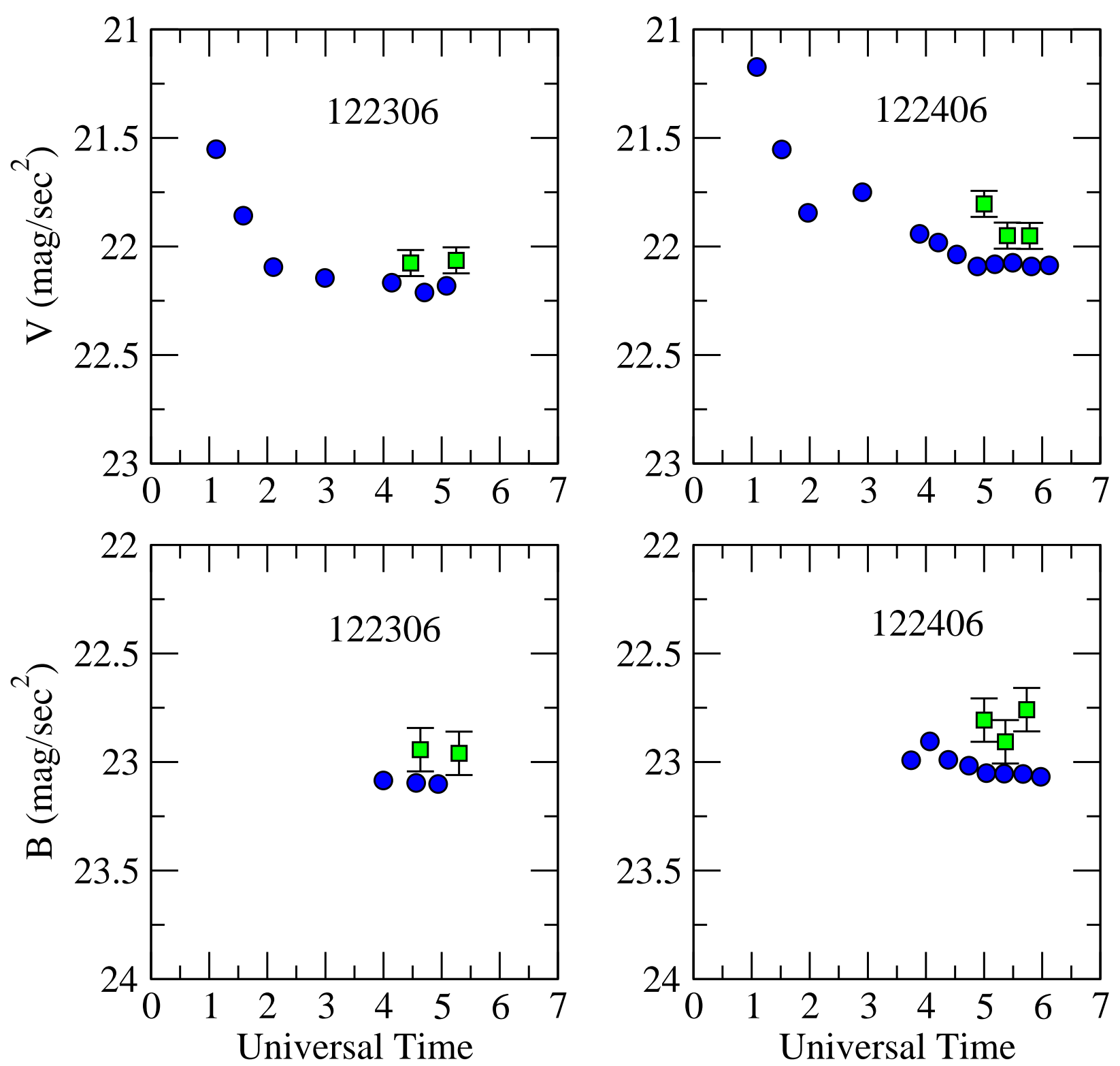

Krisciunas et al. Fig. 1 


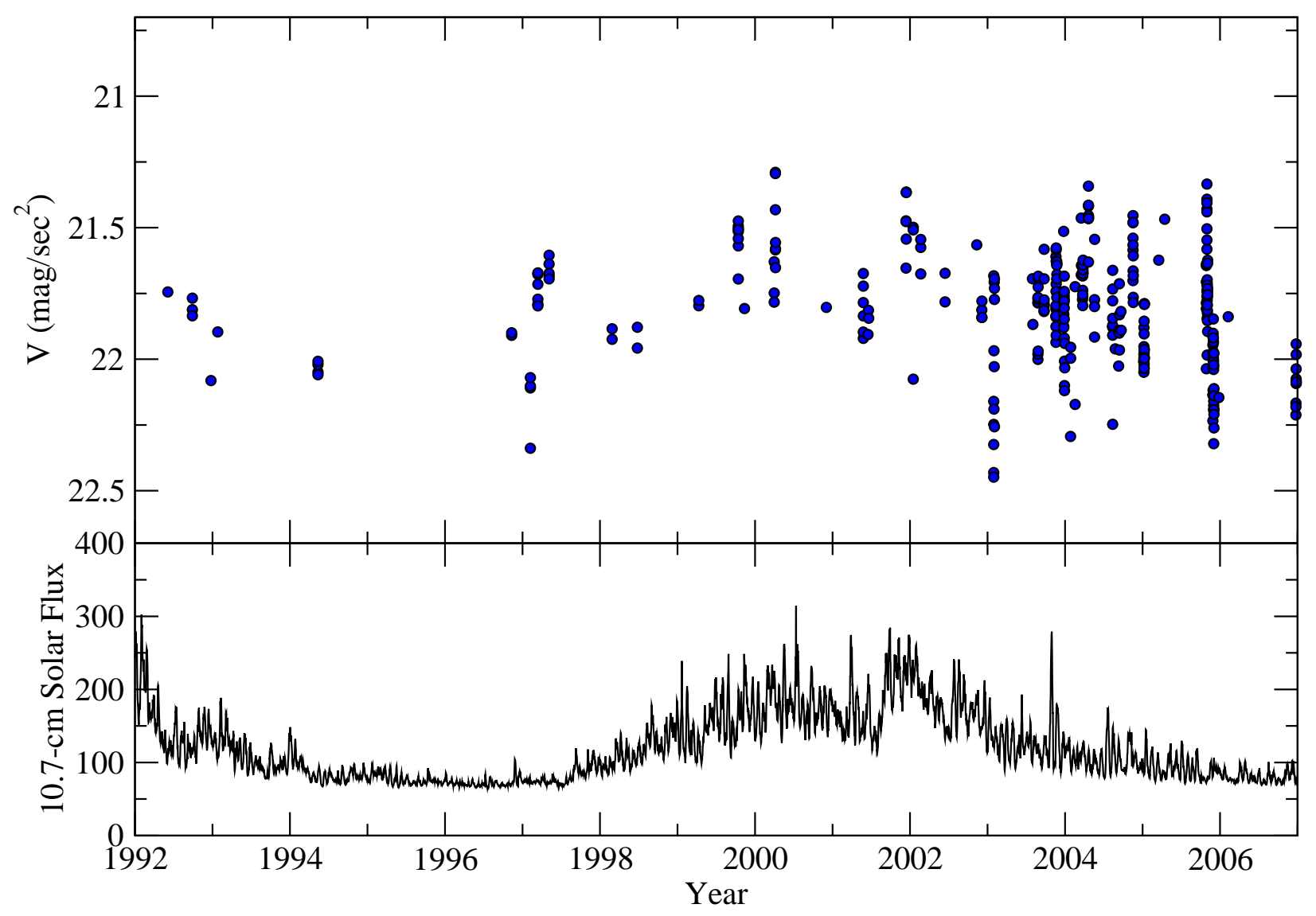

Krisciunas et al. Fig. 2 


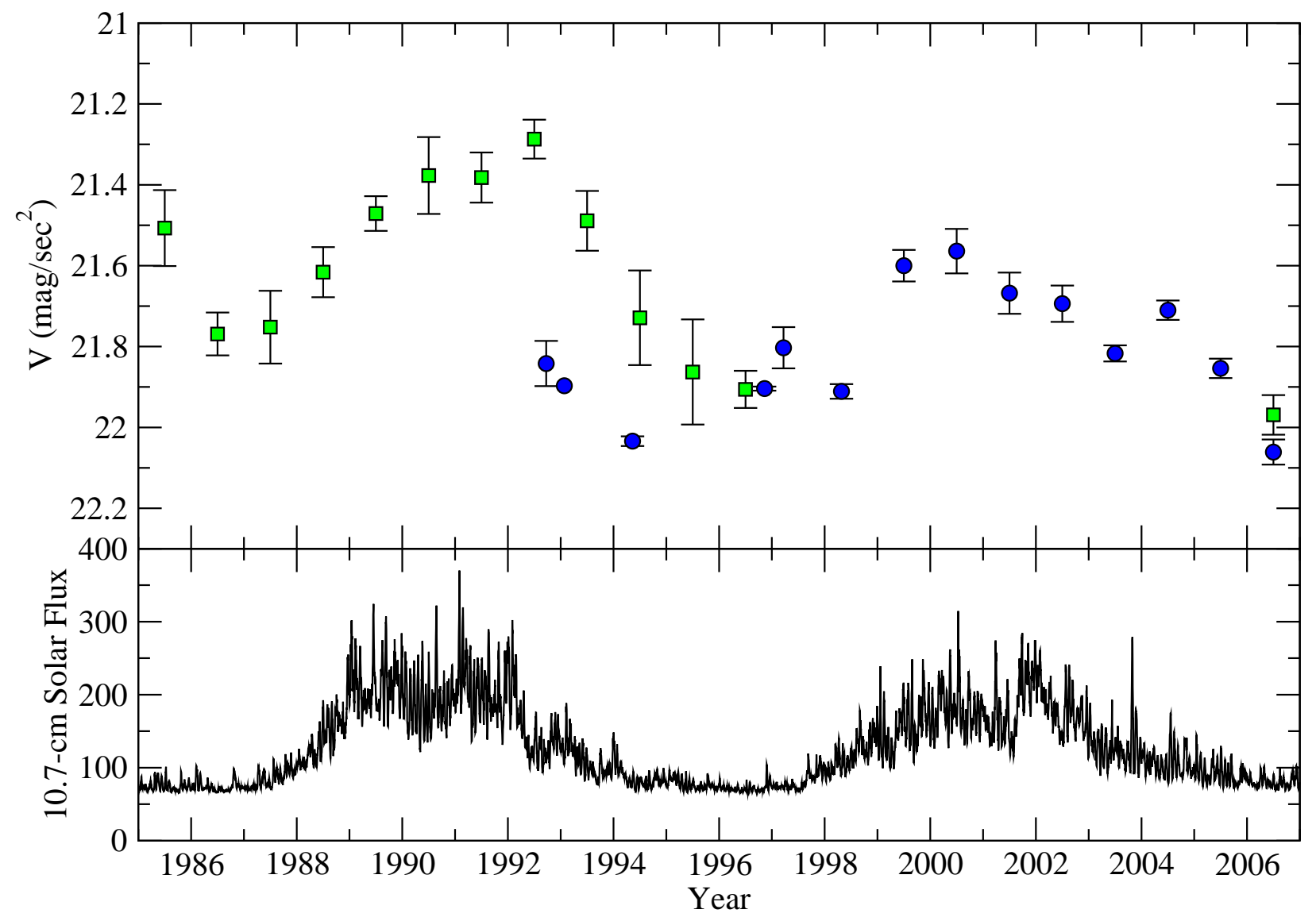

Krisciunas et al. Fig. 3 


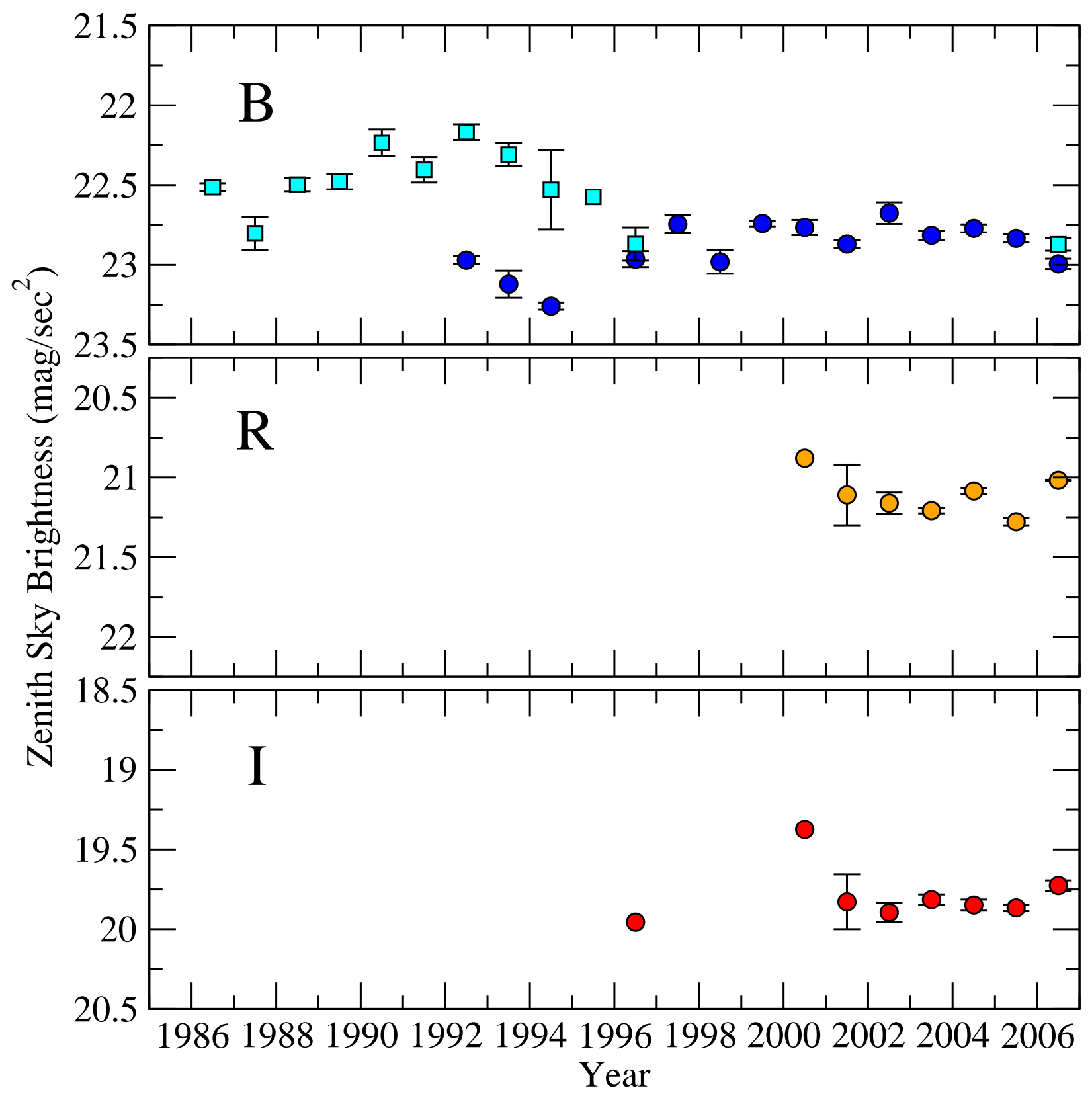

Krisciunas et al. Fig. 4 


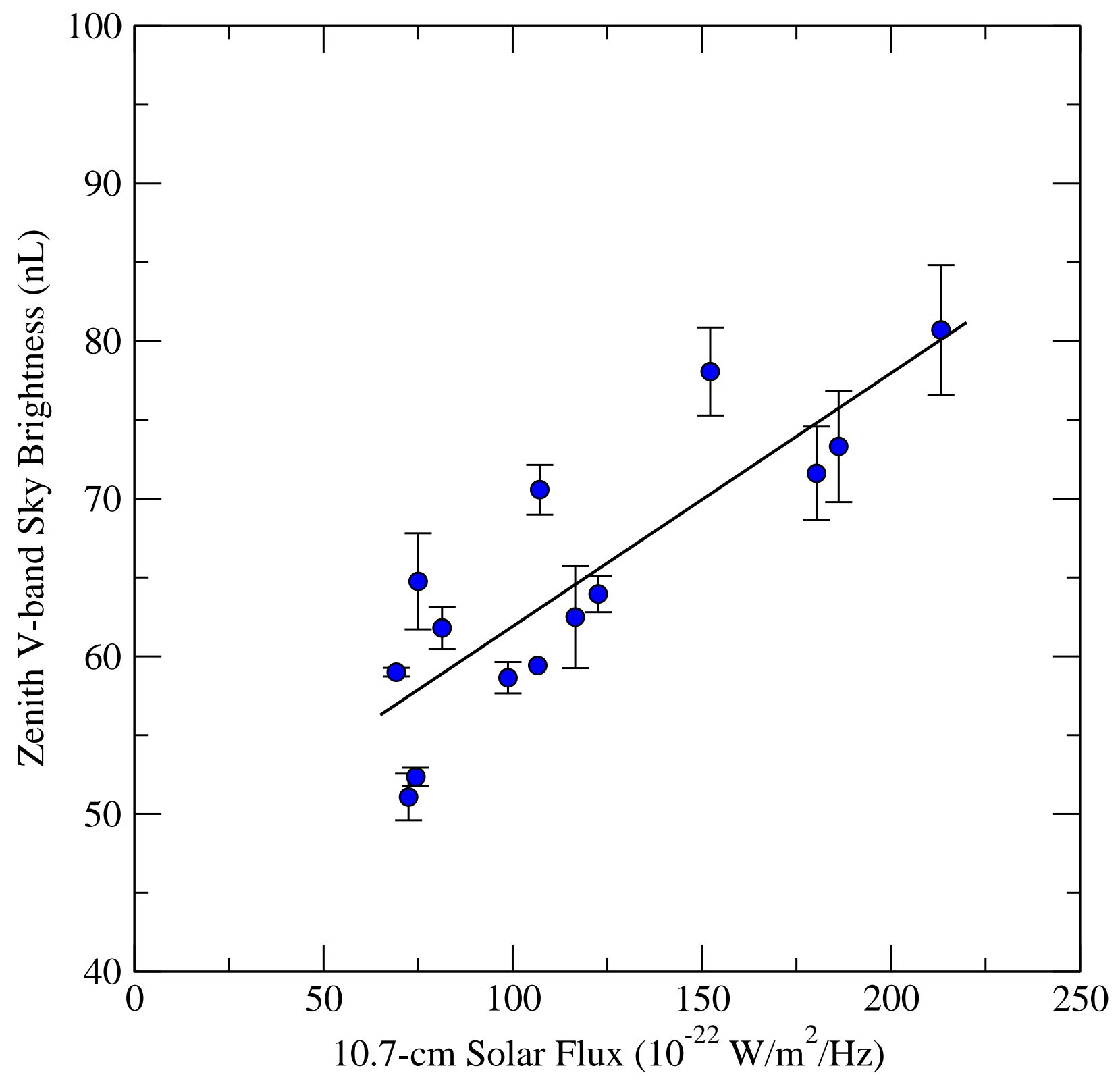

Krisciunas et al. Fig. 5 